\section{Acompanhamento do crescimento em crianças menores de um ano: situação nos serviços de saúde em Pernambuco, Brasil}

\author{
Monitoring of infant growth by health services in \\ Pernambuco State, Brazil
}

\author{
1 Departamento de Nutrição, \\ Universidade Federal de \\ Pernambuco, Recife, Brasil. \\ 2 Faculdade de Medicina, \\ Universidade Federal de \\ Pelotas, Pelotas, Brasil. \\ 3 Secretaria Estadual de \\ Saúde de Pernambuco, \\ Recife, Brasil. \\ 4 Instituto Materno Infantil \\ Professor Fernando Figueira, \\ Recife, Brasil. \\ Correspondência \\ M. F. Carvalho \\ Programa de Pós-graduação \\ em Nutrição, Departamento \\ de Nutrição, Universidade \\ Federal de Pernambuco. \\ Av. Moraes Rego s/n, Cidade \\ Universitária, Recife, $P E$ \\ 50670-901, Brasil. \\ michelledcf@hotmail.com
}

\begin{abstract}
A situational diagnosis was performed on infant growth monitoring in Greater Metropolitan Recife and in the interior of the State of Pernambuco, Brazil, using the database from the Survey on Maternal and Child Health in the State of Pernambuco, 1998, which included 816 infants (< 1 year of age) and 120 health units. Epi Info version 6.04 was used for the analysis. In the health services infrastructure, $15.8 \%$ of the health units lacked infant scales and the Child's Health Card, and $75.4 \%$ lacked the guidelines for monitoring growth and development. Of these variables, the first two showed a difference between the two study areas. As for process evaluation, $81.2 \%$ of the mothers had the Child's Health Card, 53.1\% of the children had been weighed, and only $21 \%$ had been measured. Mothers had received insufficient orientation. These variables also showed differences between the two study areas. Care for children with nutritional risk received little attention in the two areas, although there was a difference between the interior and Metropolitan Recife. In conclusion, monitoring of infant growth has not been effectively consolidated in the State of Pernambuco.
\end{abstract}

Growth; Child Welfare; Health Services Evaluation

\author{
Michelle Figueiredo Carvalho 1 \\ Pedro Israel Cabral de Lira 1 \\ Sylvia de Azevedo Mello Romani 1 \\ Iná S. Santos 2 \\ Ana Amélia Corrêa de Araújo Veras 3 \\ Malaquias Batista Filho 4
}

\section{Introdução}

A ação primária de acompanhamento do crescimento e desenvolvimento constitui o eixo central do cuidado infantil e é essencial para a articulação de atividades de prevenção e intervenção 1 . O Ministério da Saúde 2, em 1984, adotou o Programa de Atenção Integral à Saúde da Criança (PAISC), o qual priorizou cinco ações básicas a fim de incrementar a capacidade resolutiva dos serviços de saúde na atenção à criança, dentre estas ações está o acompanhamento do crescimento e desenvolvimento. Por meio do acompanhamento do crescimento se faz a avaliação periódica do ganho de peso e é possível avaliar o progresso da criança, identificando aquelas em maior risco de morbimortalidade, prevenindo precocemente a desnutrição e promovendo o crescimento infantil.

Assim, toda criança até os seis anos deve possuir o Cartão da Criança, o qual deve ficar com a mãe e ser levado em todas as consultas para acompanhamento do peso no gráfico e para anotações sobre a saúde e a nutrição da criança. Para avaliação do crescimento são necessárias pesagens periódicas, com o peso ao nascer representando o primeiro ponto marcado no gráfico e os demais registros conforme o calendário mínimo de consultas da criança. Cada vez que a criança comparecer ao serviço para consulta, seja por doença ou por visita programada, deve-se pesar, registrar no gráfico do cartão e traçar a curva de 
peso. Sempre que possível, deve ser medido o comprimento. As mães devem ser orientadas quanto ao calendário de vacinas, quanto à nutrição e aleitamento ${ }^{2}$.

A partir de 1995, o Ministério da Saúde adota no Brasil a estratégia AIDPI (Atenção Integral às Doenças Prevalentes da Infância), a qual apresenta um novo enfoque de abordagem à saúde da criança no primeiro nível de assistência. Essa estratégia considera os principais problemas de saúde das crianças, promovendo ações integradas curativas com medidas de prevenção e promoção. Essa ação começou a ser implantada em Pernambuco no ano de 1996 nos municípios com taxas elevadas de mortalidade infantil 3.

O presente trabalho objetivou analisar a ação de acompanhamento do crescimento de crianças menores de um ano, em unidades de saúde do Estado de Pernambuco, Brasil, em 1998, quanto à estrutura e processo ${ }^{4}$, comparando os resultados encontrados em áreas geográficas distintas do estado: Região Metropolitana do Recife e interior.

\section{Material e métodos}

O presente estudo foi realizado com base no banco de dados da pesquisa Atenção à Saúde Materno-Infantil no Estado de Pernambuco 5, desenvolvida no período de outubro a dezembro de 1998. Caracterizou-se por um estudo do tipo transversal abrangendo unidades de saúde da rede pública, municipalizada ou não, de caráter ambulatorial, e a demanda de crianças menores de 5 anos atendidas nestas unidades localizadas em 18 municípios do estado (Região Metropolitana do Recife e interior) previamente selecionados para a I e II Pesquisas Estaduais de Saúde e Nutrição (PESN) 6. Considerou-se como elemento amostral básico as unidades de saúde representadas por postos, centros, casas de parto, unidades mistas, hospitais regionais, além das unidades do Programa Saúde da Família (PSF) e do Programa de Saúde em Casa, entendendose a unidades de saúde como um conglomerado operacional de atendimento. A amostra foi do tipo probabilística (aleatória estratificada) contemplando as unidades de níveis hierárquicos 1 e 2 . As referidas unidades foram identificadas usando-se o cadastro no SIA/SUS (Sistema de Informação Ambulatorial do Sistema Único de Saúde) da Secretaria de Saúde do Estado de Pernambuco, perfazendo um total de 1.228 , sendo 221 na Região Metropolitana do Recife e 1.007 no interior do estado. Levando-se em conta o caráter de complementariedade do estudo, foram considerados como pré-selecionados os $18 \mathrm{mu}$ - nicípios investigados nas I e II PESN; o total de unidades nestes municípios correspondia a 252 . Dessas, foram estudadas aproximadamente 50\%, ou seja, 130 unidades (73 da Região Metropolitana do Recife e 57 do interior).

A seleção da unidade amostral (unidades de saúde) foi feita de forma aleatória. Para caracterizar as unidades foram entrevistados os diretores (69 da Região Metropolitana do Recife e 51 do interior), tendo sido excluídas 10 por motivo de ausência dos referidos diretores, totalizando 120 unidades pesquisadas para obtenção dos aspectos estruturais dos serviços. Ao chegar à unidade, realizava-se a entrevista com o responsável (diretor ou administrador), ao mesmo tempo em que se solicitava permissão para a realização do trabalho junto às mães ou acompanhantes das crianças menores de 5 anos que foram receber algum tipo de atendimento naquele local. Essas mães ou responsáveis eram entrevistados logo após o atendimento, sempre no horário de funcionamento da unidade. Quando a unidade estava com suas atividades encerradas ou temporariamente suspensas procedia-se a sua substituição por outra do mesmo nível de hierarquia, no mesmo município.

Apesar de informados sobre a pesquisa, os secretários e as unidades foram informados da sua inclusão na pesquisa apenas no momento da coleta dos dados, a fim de evitar interferências que influenciassem na avaliação. Para essa coleta foram utilizados como instrumentos cinco formulários e, dentre eles, um referente à criança e outro à unidade de saúde, cada qual possuindo um manual de instruções para orientação e padronização do preenchimento. Para a realização do estudo foi obtida aprovação do Conselho de Ética do Instituto Materno-Infantil de Pernambuco (IMIP), bem como o consentimento das mães ou acompanhantes para participarem das entrevistas. Os entrevistadores foram treinados por meio de aulas expositivas sobre o modelo assistencial/SUS e atendimento integrado à criança, bem como através de discussões dos formulários, dramatizações e aplicação prática dos formulários no ambulatório do IMIP. Os questionários eram revisados na presença do entrevistado. Em seguida os questionários passavam por mais duas revisões pelos supervisores e equipe técnica do Departamento de Nutrição da Universidade Federal de Pernambuco.

Para a análise do banco de dados, primeiramente foram selecionadas as crianças menores de 1 ano, totalizando 816 crianças. A seguir, foram realizadas análises utilizando-se o programa Epi Info versão 6.04 (Centers for Disease Control and Prevention, Atlanta, Estados Unidos). Procedeu-se a um diagnóstico situacional da ação de 
acompanhamento do crescimento nas unidades de saúde de áreas geográficas distintas no que diz respeito à estrutura e processo.

Para efeito das análises utilizaram-se as variáveis especificadas a seguir: (a) relacionadas ao espaço geográfico - Região Metropolitana do Recife (abrangendo cinco municípios) e interior do estado (13 municípios); (b) relacionadas às crianças menores de 1 ano - idade, sexo, peso ao nascer, estado nutricional (relação peso/idade, com base na posição do peso atual registrado no cartão), cuidados com a criança, motivos da ida à unidade de saúde; (c) relacionadas às mães - idade (em anos), nível de instrução; (d) relacionadas às condições sócio-econômicas das famílias - renda per capita; (e) relacionadas à estrutura dos serviços - existência de balança pesa-bebê, de Cartão da Criança, de normas do Ministério da Saúde para o acompanhamento do crescimento e do desenvolvimento, e de profissionais sem treinamento para atendimento à criança; (f) relacionadas ao processo de acompanhamento do crescimento da criança nas unidades de saúde - pesagem, medição do comprimento, posse do cartão, registro do peso ao nascer no cartão, pontuação do peso ao nascer no gráfico, registro do peso atual no gráfico (crianças pesadas no dia da entrevista e que portavam o Cartão da Criança), informações prestadas às mães sobre o crescimento da criança (peso, comprimento e ponto do peso no gráfico), conduta relativa ao monitoramento do crescimento na primeira consulta (pesagem, medidas de comprimento, peso atual marcado no gráfico e se as mães receberam orientação sobre o crescimento); (g) relacionadas às crianças sob risco nutricional (baixo peso e peso insuficiente ao nascer, direção de curva de crescimento descendente e horizontal, peso abaixo do percentil 10 e acima do percentil 90) - pontuação do peso ao nascer no gráfico, pesagem, peso atual no gráfico (crianças pesadas no dia da entrevista e que portavam o Cartão da Criança), medição do comprimento, orientação às mães sobre a direção da curva do peso, orientação sobre a posição do ponto do peso atual.

\section{Resultados}

Do total de 1.611 crianças da pesquisa Atenção à Saúde Materno-Infantil no Estado de Pernambuco, 816 menores de 1 ano de idade foram incluídas no estudo. Na Tabela 1 estão descritas as características das crianças, estratificadas segundo o local de residência. Não houve diferença estatisticamente significante entre as duas áreas quanto à distribuição de idade, do sexo e do peso ao nascer das crianças, como também, com re- lação aos cuidados com a criança e à idade das mães. Do total de crianças que tinham o registro do peso ao nascer no cartão (592), 8,2\% nasceram com baixo peso $(<2.500 \mathrm{~g})$ e $20,9 \%$ com peso insuficiente (2.500g-2.999g). Em relação aos dois estratos, 9,7\% e 5,6\% na Região Metropolitana do Recife e interior, respectivamente, nasceram com baixo peso. A grande maioria das crianças (96\%) era cuidada pela mãe biológica. As mães adolescentes ( $<20$ anos) e as de 30 anos e mais de idade representavam $25,1 \%$ e $16,9 \%$, respectivamente. Houve um leve predomínio de mães adolescentes na Região Metropolitana do Recife $(28,1 \%)$ em relação às do interior $(21,4 \%)$.

De acordo com a posição do ponto do peso atual das crianças no cartão, aferido no dia da consulta, em nível do Estado de Pernambuco, $6,8 \%$ delas encontravam-se com peso para idade abaixo do percentil 10 e 14,5\% acima do percentil 90. A diferença entre os estratos foi estatisticamente significativa $(p=0,01)$, sendo que a prevalência de crianças abaixo do percentil 10 foi cerca de duas vezes mais freqüente no interior do que na Região Metropolitana do Recife (9,6\% e 5,2\%, respectivamente). A prevalência de crianças acima do percentil 90, ao contrário, foi três vezes superior na Região Metropolitana do Recife.

Em relação à visita ao serviço de saúde, 40,8\% das crianças no estado compareceram por motivo de vacinação, constituindo-se também o principal motivo de ambas as áreas. A doença foi o segundo motivo referido no interior $(37,6 \%)$. Na Região Metropolitana do Recife, o segundo motivo foi a visita de rotina (31,3\%), enquanto que, no interior, apenas $5,8 \%$ compareceram ao serviço com esta finalidade. A diferença entre os dois grupos quanto ao motivo da consulta foi também estatisticamente significativa, tendo as crianças da Região Metropolitana do Recife sido levadas às unidades de saúde para consultas de rotina cerca de seis vezes mais freqüentemente do que as do interior $(p<0,001)$.

Em relação ao nível de instrução das mães ou responsáveis pelas crianças, $11,7 \%$ eram analfabetas e a grande maioria tinha o $1^{\circ}$ grau incompleto $(61,1 \%)$. A diferença de escolaridade entre os dois grupos foi estatisticamente significante ( $p=0,003$ ), tendo-se verificado no interior um percentual de mães analfabetas quase duas vezes maior do que o observado na Região Metropolitana (respectivamente, 15,9\% e 8,4\%). Do mesmo modo, a análise da renda revelou diferença significante $(\mathrm{p}=0,02)$, encontrando-se na primeira categoria $(<0,25$ do salário mínimo) freqüência maior no interior $(33,6 \%)$ do que na Região Metropolitana do Recife $(23,8 \%)$.

Na Tabela 2, a análise da estrutura dos serviços com base nas informações fornecidas pe- 
Tabela 1

Caracterização das crianças, das mães e das famílias segundo aspectos biológicos, demográficos e sócio-econômicos de acordo com a localização do serviço de saúde. Pernambuco, Brasil, 1998.

\begin{tabular}{|c|c|c|c|c|c|c|c|}
\hline \multirow[t]{2}{*}{ Variáveis } & \multicolumn{2}{|c|}{$\begin{array}{l}\text { Estado } \\
(\mathrm{N}=816)\end{array}$} & \multicolumn{2}{|c|}{$\begin{array}{l}\text { Região Metropolitana do Recife } \\
\qquad(\mathrm{N}=454)\end{array}$} & \multicolumn{2}{|c|}{$\begin{array}{l}\text { Interior } \\
(\mathrm{N}=362)\end{array}$} & \multirow[t]{2}{*}{$p$ * } \\
\hline & $\mathrm{n}$ & $\%$ & $\mathbf{n}$ & $\%$ & $\mathbf{n}$ & $\%$ & \\
\hline \multicolumn{8}{|l|}{ Das crianças } \\
\hline Idade (meses) & & & & & & & 0,2 \\
\hline$<6$ & 453 & 55,5 & 260 & 57,3 & 193 & 53,3 & \\
\hline $6-11,9$ & 363 & 44,5 & 194 & 42,7 & 169 & 46,7 & \\
\hline Sexo & & & & & & & 0,8 \\
\hline Masculino & 423 & 51,8 & 234 & 51,5 & 189 & 52,2 & \\
\hline Feminino & 393 & 48,2 & 220 & 48,5 & 173 & 47,8 & \\
\hline Peso ao nascer em gramas $(n=592) \star \star$ & & & & & & & 0,1 \\
\hline$<2.500$ & 48 & 8,2 & 35 & 9,7 & 13 & 5,6 & \\
\hline $2.500-2.999$ & 124 & 20,9 & 79 & 21,8 & 45 & 19,6 & \\
\hline$\geq 3.000$ & 420 & 70,9 & 248 & 68,5 & 172 & 74,8 & \\
\hline 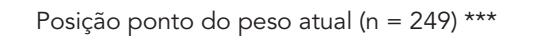 & & & & & & & 0,01 \\
\hline Acima do percentil 90 & 36 & 14,5 & 30 & 19,3 & 6 & 6,4 & \\
\hline Entre percentil 10 e percentil 90 & 196 & 78,7 & 117 & 75,5 & 79 & 84,0 & \\
\hline Abaixo do percentil 10 & 17 & 6,8 & 8 & 5,2 & 9 & 9,6 & \\
\hline \multicolumn{8}{|l|}{ Cuidados com a criança } \\
\hline Mãe biológica & 785 & 96,2 & 437 & 96,3 & 348 & 96,1 & 0,9 \\
\hline Outra & 31 & 3,8 & 17 & 3,7 & 14 & 3,9 & \\
\hline Motivo da ida à unidade & & & & & & & $<0,001$ \\
\hline Vacinação & 333 & 40,8 & 158 & 34,8 & 175 & 48,3 & \\
\hline Rotina & 163 & 20,0 & 142 & 31,3 & 21 & 5,8 & \\
\hline Doença/Retorno & 269 & 33,0 & 133 & 29,3 & 136 & 37,6 & \\
\hline Suplementação alimentar/Outro & 51 & 6,2 & 21 & 4,6 & 30 & 8,3 & \\
\hline \multicolumn{8}{|l|}{ Das mães ou responsáveis } \\
\hline Idade materna em anos $(n=812) \#$ & & & & & & & 0,07 \\
\hline$<19$ & 204 & 25,1 & 127 & 28,1 & 77 & 21,4 & \\
\hline $20-24$ & 287 & 35,3 & 162 & 35,8 & 125 & 34,7 & \\
\hline $25-29$ & 184 & 22,7 & 95 & 21,0 & 89 & 24,7 & \\
\hline$\geq 30$ & 137 & 16,9 & 68 & 15,1 & 69 & 19,2 & \\
\hline Nível de instrução $(n=813) \# \#$ & & & & & & & 0,003 \\
\hline Analfabeta & 95 & 11,7 & 38 & 8,4 & 57 & 15,9 & \\
\hline 1ㅇ grau incompleto & 497 & 61,1 & 282 & 62,2 & 215 & 59,7 & \\
\hline 1ㅇ grau completo e/ou 2o grau incompleto & 124 & 15,3 & 80 & 17,7 & 44 & 12,2 & \\
\hline 2o grau completo e/ou superior & 97 & 11,9 & 53 & 11,7 & 44 & 12,2 & \\
\hline \multicolumn{8}{|l|}{ Das famílias } \\
\hline Renda per capita em salários mínimos $(n=68$ & & & & & & & 0,02 \\
\hline$<0,25$ & 196 & 28,4 & 87 & 23,8 & 109 & 33,6 & \\
\hline $0,25-0,49$ & 237 & 34,4 & 129 & 35,3 & 108 & 33,4 & \\
\hline $0,50-1,00$ & 175 & 25,4 & 105 & 28,8 & 70 & 21,6 & \\
\hline$\geq 1,00$ & 81 & 11,8 & 44 & 12,1 & 37 & 11,4 & \\
\hline
\end{tabular}

* $\mathrm{p}$ da diferença entre a Região Metropolitana do Recife e interior;

** Total de 592 crianças, pois 224 não tinham o registro do peso ao nascer no cartão (78 da Região Metropolitana do Recife e 146 do interior);

*** Das 816 crianças, apenas 249 tinham a posição do peso atual assinalado no cartão;

\# Quatro idades ignoradas (duas na Região Metropolitana do Recife e duas no interior);

\#\# Três mães não revelaram o nível de instrução (uma da Região Metropolitana do Recife e duas no interior);

\#\#\# Renda calculada com base no salário mínimo da época (R\$130,00), 127 famílias não informaram a renda total não sendo possível incluí-las no cálculo da renda per capita (89 da Região Metropolitana do Recife e 38 do interior). 
Características relacionadas à estrutura das unidades de saúde para o acompanhamento do crescimento das crianças nos serviços de saúde, segundo a localização. Pernambuco, Brasil, 1998.

\begin{tabular}{|c|c|c|c|c|c|c|c|}
\hline \multirow[t]{2}{*}{ Variáveis } & \multicolumn{2}{|c|}{$\begin{array}{l}\text { Estado } \\
(\mathrm{N}=816)\end{array}$} & \multicolumn{2}{|c|}{$\begin{array}{l}\text { Região Metropolitana do Recife } \\
\qquad(\mathrm{N}=454)\end{array}$} & \multicolumn{2}{|c|}{$\begin{array}{l}\text { Interior } \\
(\mathrm{N}=362)\end{array}$} & \multirow[t]{2}{*}{$p$ * } \\
\hline & $\mathbf{n}$ & $\%$ & $\mathbf{n}$ & $\%$ & $\mathrm{n}$ & $\%$ & \\
\hline Balança pesa-bebê & & & & & & & 0,04 \\
\hline Sim, funcionando & 101 & 84,2 & 62 & 89,8 & 39 & 76,5 & \\
\hline Sim, não funcionando/Não tem & 19 & 15,8 & 7 & 10,2 & 12 & 23,5 & \\
\hline Cartão da criança & & & & & & & 0,04 \\
\hline Sim, suficiente & 101 & 84,2 & 62 & 89,8 & 39 & 76,5 & \\
\hline Não/Insuficiente & 19 & 15,8 & 7 & 10,2 & 12 & 23,5 & \\
\hline Normas do programa $(n=114) * \star$ & & & & & & & 0,3 \\
\hline Sim & 28 & 24,6 & 14 & 21,5 & 14 & 28,6 & \\
\hline Não & 86 & 75,4 & 51 & 78,5 & 35 & 71,4 & \\
\hline \multicolumn{8}{|l|}{ Profissionais não treinados } \\
\hline Médicos $(n=211)$ & 159 & 75,3 & 84 & 76,4 & 75 & 74,2 & 0,7 \\
\hline Enfermeiros $(n=82)$ & 42 & 51,2 & 27 & 50,0 & 15 & 53,6 & 0,7 \\
\hline Auxiliares de enfermagem $(n=231)$ & 182 & 78,8 & 113 & 84,3 & 69 & 71,1 & 0,01 \\
\hline Nutricionistas $(\mathrm{n}=10)$ & 6 & 60,0 & 1 & 100,0 & 5 & 55,5 & $1,0 * \star \star$ \\
\hline Atendentes $(n=385)$ & 262 & 68,0 & 174 & 65,9 & 88 & 72,7 & 0,1 \\
\hline
\end{tabular}

* $p$ da diferença entre Região Metropolitana do Recife e interior;

** Seis unidades de saúde estavam sem informação quanto às normas (quatro da Região Metropolitana do Recife e duas do interior);

*** Teste exato de Fischer.

los respectivos diretores, revelou que $84,2 \%$ das unidades pesquisadas dispunham de balança pesa-bebê funcionando, e em 15,8\% delas, este equipamento não funcionava ou não existia. Na Região Metropolitana do Recife, essa situação foi observada em 10,2\% das unidades de saúde e em 23,5\% das do interior. Quanto ao Cartão da Criança, estava disponível e era suficiente em cerca de $80 \%$ das unidades do estado. No interior, $23,5 \%$ não possuíam esse instrumento ou era insuficiente, contra $10,2 \%$ na região metropolitana. Mais de $70 \%$ das unidades de ambos os estratos analisados, não apresentaram as normas para o acompanhamento do crescimento e desenvolvimento infantis. Os testes estatísticos não revelaram diferenças significantes entre as duas áreas, exceto para a presença de balança pesa-bebê ( $p$ $=0,04)$ e Cartão da Criança $(\mathrm{p}=0,04)$.

Verificou-se que mais de $70 \%$ dos médicos responsáveis pelo atendimento às crianças declararam não haver recebido treinamento específico para as ações de assistência à saúde das mesmas. Entre os demais profissionais, encontraram-se percentuais de $51,2 \%, 78,8 \%$ e $68 \%$, respectivamente, de enfermeiros, auxiliares de enfermagem e atendentes não treinados no estado. Chama atenção o reduzido número de profis- sionais nutricionistas (apenas dez) como membro da equipe de saúde, e a elevada proporção de auxiliares de enfermagem nas unidades de saúde das duas áreas (84,3\% e 71,1\%, respectivamente) que declararam não haver recebido treinamento específico para o atendimento às crianças. Não foi observada diferença estatisticamente significante em relação a essa variável, com exceção dos auxiliares de enfermagem que diferiram significativamente nas duas áreas $(\mathrm{p}=0,01)$.

No que diz respeito ao processo de acompanhamento do crescimento das crianças nas unidades de saúde (Tabela 3), observou-se que aproximadamente metade das que foram atendidas havia sido pesada no dia da entrevista, com maior freqüência na Região Metropolitana do Recife $(58,8 \%)$ do que no interior (46\%). Apenas $21 \%$ das crianças haviam sido medidas em comprimento, sendo esta prática duas vezes menos freqüente no interior do que na Região Metropolitana. Dentre o total de crianças estudadas, $81,2 \%$ apresentaram o cartão no momento da entrevista, embora no interior $28,1 \%$ das mães não o tinham levado para a consulta, contra $11,3 \%$ na Região Metropolitana do Recife. Essas variáveis diferiram estatisticamente entre as duas áreas $(\mathrm{p}<0,001)$. 
Características relacionadas ao processo de acompanhamento do crescimento das crianças nos serviços de saúde, segundo a localização. Pernambuco, Brasil, 1998

\begin{tabular}{|c|c|c|c|c|c|c|c|}
\hline \multirow[t]{2}{*}{ Variáveis } & \multicolumn{2}{|c|}{ Estado } & \multicolumn{2}{|c|}{ Região Metropolitana do Recife } & \multicolumn{2}{|c|}{ Interior } & \multirow[t]{2}{*}{ p * } \\
\hline & $\mathbf{n}$ & $\%$ & $\mathbf{n}$ & $\%$ & $\mathbf{n}$ & $\%$ & \\
\hline 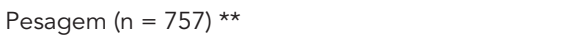 & & & & & & & $<0,001$ \\
\hline Sim & 402 & 53,1 & 247 & 58,8 & 155 & 46,0 & \\
\hline Medição do comprimento $(\mathrm{n}=757)$ ** & & & & & & & $<0,001$ \\
\hline Sim & 159 & 21,0 & 112 & 26,7 & 47 & 13,9 & \\
\hline 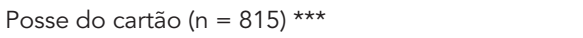 & & & & & & & $<0,001$ \\
\hline Sim, trouxe & 662 & 81,2 & 402 & 88,7 & 260 & 71,9 & \\
\hline Sim, não trouxe & 123 & 15,1 & 40 & 8,9 & 83 & 22,9 & \\
\hline Não tem & 30 & 3,7 & 11 & 2,4 & 19 & 5,2 & \\
\hline Registro do peso ao nascer no cartão $(n=662) \#$ & & & & & & & 0,5 \\
\hline Sim & 592 & 89,4 & 362 & 90,0 & 230 & 88,5 & \\
\hline Pontuação do peso ao nascer no gráfico $(n=662)$ \# & & & & & & & 0,1 \\
\hline Sim & 261 & 44,1 & 168 & 46,4 & 93 & 40,4 & \\
\hline Peso atual no gráfico $(n=402) \# \#$ & & & & & & & 0,8 \\
\hline $\operatorname{Sim}$ & 234 & 58,2 & 143 & 57,9 & 91 & 58,7 & \\
\hline Informações dadas às mães ( $n=333$ ) & & & & & & & 0,8 \\
\hline Peso & 143 & 35,5 & 119 & 48,0 & 24 & 15,5 & \\
\hline Comprimento & 85 & 53,5 & 73 & 65,2 & 12 & 25,5 & \\
\hline Ponto do peso marcado no gráfico & 105 & 43,2 & 87 & 58,0 & 18 & 19,4 & \\
\hline Primeira consulta $(n=232)$ & & & & & & & 0,01 \\
\hline Pesagem & 102 & 54,3 & 58 & 64,4 & 44 & 44,9 & \\
\hline Medição do comprimento & 38 & 20,2 & 28 & 31,1 & 10 & 10,2 & \\
\hline Peso atual marcado no gráfico & 52 & 22,4 & 28 & 19,2 & 24 & 27,9 & \\
\hline Orientação sobre o crescimento & 40 & 21,5 & 32 & 36,4 & 8 & 8,2 & \\
\hline
\end{tabular}

* p da diferença entre Região Metropolitana do Recife e interior;

** Considerando apenas as crianças que receberam atendimento no dia da entrevista;

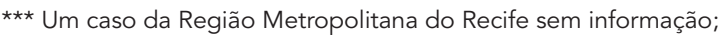

\# Considerando apenas aquelas que apresentaram o cartão no dia da entrevista;

\#\# Considerando apenas as crianças que foram pesadas no dia da entrevista.

Aproximadamente $90 \%$ das 662 crianças cujas mães portavam o cartão no momento da entrevista, em ambos os estratos, tinham o registro do peso ao nascer, porém, menos da metade tinha este peso pontuado no gráfico. No Estado de Pernambuco, de um total de 402 crianças que haviam sido pesadas no dia da entrevista, 234 $(58,2 \%)$ tiveram o peso marcado no gráfico, valores semelhantes aos encontrados nos dois estratos, não sendo observadas, portanto, diferenças estatisticamente significantes entre eles.

Ainda com relação ao processo de acompanhamento do crescimento infantil, detectou-se que percentuais reduzidos de mães foram informadas sobre aspectos do crescimento dos seus filhos como peso, altura e situação do peso no gráfico, nas consultas realizadas nas unidades de saúde. Embora no interior as freqüências te- nham sido mais baixas (15,5\%; $25,5 \%$ e $19,4 \%)$ do que na Região Metropolitana do Recife (48\%; $65,2 \%$ e $58 \%$ ), não se observaram diferenças estatisticamente significantes.

O mesmo ocorreu com relação à primeira consulta das crianças, cujas avaliações do crescimento por meio da pesagem, da medição do comprimento e da marcação do peso atual no gráfico para posterior avaliação da tendência do crescimento, foram bastante reduzidas, embora mais freqüentes na Região Metropolitana do Recife, chegando a superar até três vezes as freqüências observadas no interior, como foi o caso da medição do comprimento. Por outro lado, o registro do peso atual no gráfico foi mais freqüente no interior (27,9\%) do que na Região Metropolitana (19,2\%). Apenas 36,4\% das mães da Região Metropolitana e 8,2\% do interior re- 
ceberam alguma orientação sobre a avaliação do crescimento dos seus respectivos filhos nessa primeira consulta. A análise estatística revelou diferenças significativas entre as duas áreas com relação ao monitoramento do crescimento na primeira consulta $(\mathrm{p}=0,01)$.

Considerando-se as crianças sob risco nutricional (de baixo peso e peso insuficiente ao nascer) (Tabela 4), cujos totais corresponderam a 172, 114 e 58 para o estado, região metropolitana e interior, respectivamente, a atenção ao seu crescimento foi bastante precária, com um percentual bastante elevado de crianças que deixaram de receber a devida atenção quanto ao seu crescimento. As "pontuações do peso ao nascer" e do "peso atual no gráfico" não atingiram os $50 \%$ das crianças, embora tenham sido mais acentuadas no interior (48,3\% e 41,4\%, respectivamente), enquanto a "medição do peso e do comprimento" ocorreu com mais freqüência na Região Metropolitana do Recife (56,1\% e 23,7\%, respectivamente). A análise estatística não revelou diferenças significativas entre as áreas com relação a esses aspectos estudados.

As crianças que apresentaram direção e posição da curva de peso desfavoráveis (Tabela 4), também consideradas como de risco nutricional, não tiveram sua condição informada às mães, as quais foram orientadas para enfrentamento do problema em número bastante reduzido, principalmente no interior, cujos percentuais corresponderam a apenas $18,2 \%$ e $20 \%$, respectivamente. Não houve diferença significativa entre as áreas no que diz respeito à orientação às mães sobre a direção da curva, porém, em relação à orientação sobre a posição do peso atual houve diferença estatisticamente significante entre as áreas rural e metropolitana, mostrando um melhor desempenho na Região Metropolitana do Recife do que no interior.

\section{Discussão}

Embora não haja evidências seguras de que a monitorização do crescimento per se tenha efeito sobre a melhoria do estado nutricional na infância, esta prática vem sendo utilizada não só no Brasil, por meio do PAISC e da estratégia AIDPI, como em vários países em desenvolvimento. Independente de impacto, a monitorização do crescimento tem a vantagem de fornecer feedback aos profissionais de saúde sobre o efeito de intervenções ou programas dirigidos à população infantil. A monitorização efetiva, no entanto, requer no mínimo um bom nível de registros,

Características relacionadas ao processo de acompanhamento do crescimento de crianças sob risco nutricional nos serviços de saúde, segundo a localização. Pernambuco, Brasil, 1998.

\begin{tabular}{|c|c|c|c|c|c|c|c|}
\hline \multirow[t]{3}{*}{ Crianças sob risco nutricional * } & \multicolumn{7}{|c|}{ Estratos geográficos } \\
\hline & \multicolumn{2}{|c|}{$\begin{array}{l}\text { Estado } \\
(\mathrm{N}=172)\end{array}$} & \multicolumn{2}{|c|}{$\begin{array}{l}\text { Região Metropolitana do Recife } \\
\qquad(\mathrm{N}=114)\end{array}$} & \multicolumn{2}{|c|}{$\begin{array}{l}\text { Interior } \\
(\mathrm{N}=58)\end{array}$} & \multirow[t]{2}{*}{$p * *$} \\
\hline & $\mathrm{n}$ & $\%$ & $\mathrm{n}$ & $\%$ & $n$ & $\%$ & \\
\hline \multicolumn{8}{|l|}{ Crianças com baixo peso $(<2.500 \mathrm{~g})$ e peso } \\
\hline insuficiente ao nascer $(2.500 \mathrm{~g}-2.999 \mathrm{~g})$ & - & - & - & - & - & - & - \\
\hline Pontuação do peso ao nascer no gráfico & 88 & 51,2 & 50 & 43,9 & 28 & 48,3 & 0,7 \\
\hline Peso atual no gráfico & 67 & 39,0 & 43 & 37,7 & 24 & 41,4 & 0,76 \\
\hline Pesagem & 88 & 51,2 & 64 & 56,1 & 24 & 41,4 & 0,1 \\
\hline Medição do comprimento & 37 & 21,5 & 27 & 23,7 & 10 & 17,2 & 0,44 \\
\hline Crianças com direção de curva descendente e horizontal & 48 & - & 37 & - & 11 & - & - \\
\hline Orientação às mães sobre direção da curva de peso & 21 & 43,8 & 19 & 51,4 & 2 & 18,2 & $0,08 * \star \star$ \\
\hline \multicolumn{8}{|l|}{ Crianças com posição de peso abaixo do percentil 10} \\
\hline e acima do percentil 90 & 53 & - & 38 & - & 15 & - & - \\
\hline Orientação às mães sobre posição do peso atual \# & 27 & 50,9 & 24 & 63,2 & 3 & 20,0 & 0,01 \\
\hline \multicolumn{8}{|c|}{$\begin{array}{l}\text { * Crianças de baixo peso e peso insuficiente ao nascer, com curvas de peso descendente e horizontal e com posição do peso abaixo do percentil } 10 \text { e acima } \\
\text { do percentil } 90 ;\end{array}$} \\
\hline \multicolumn{8}{|l|}{${ }^{\star *} \mathrm{p}$ da diferença entre Região Metropolitana do Recife e interior; } \\
\hline \multicolumn{8}{|l|}{ *** Teste exato de Fischer; } \\
\hline \# Relação peso para idade. & & & & & & & \\
\hline
\end{tabular}


altas taxas de cobertura, regularidade do atendimento, mensurações corretas das medidas antropométricas, profissionais treinados, educação materna nas ações de saúde e nutrição e adequada intervenção ?

Essa avaliação da estrutura e do processo da ação de acompanhamento do crescimento no Estado de Pernambuco mostrou que há importantes deficiências que necessitam ser consideradas. Em termos estruturais, faltam balanças, Cartão da Criança, normas do programa e treinamento de profissionais em uma substancial proporção de unidades de saúde, particularmente no interior do estado. A balança representa a factibilidade da ação, ou seja, sem a balança torna-se inviável obter o peso da criança e assim, promover as etapas subseqüentes do processo (marcação no cartão, diagnóstico do estado nutricional, aconselhamento, tratamento e promoção da saúde). No que diz respeito a esse recurso, encontrou-se para o estado uma carência de aproximadamente $16 \%$, com maior prejuízo para o interior e, como conseqüência, cerca de metade das crianças não foi pesada no dia da entrevista.

O Cartão da Criança é um instrumento de acompanhamento da saúde, nutrição, crescimento e desenvolvimento na infância, sendo importante para a participação das mães neste processo. No presente estudo, o estoque de Cartão da Criança foi considerado suficiente em mais de $90 \%$ das unidades de saúde. Segundo Ghosh 8 o estoque regular, suficiente, a manutenção e reposição dos cartões, balanças e outros itens são fundamentais para o sucesso do acompanhamento do crescimento. A existência das normas do Ministério da Saúde para o acompanhamento do crescimento e desenvolvimento estava em apenas 1/4 das unidades pesquisadas. As normas são essenciais para serem consultadas em caso de dúvidas e servirem de material para procedimentos de treinamento.

Verificou-se um elevado percentual de profissionais não treinados nas ações de assistência às crianças nos estratos geográficos estudados, bem como reduzido número do profissional nutricionista como membro da equipe de saúde. Isso demonstra não apenas a necessidade de treinamento, mas também a falta de equipe multiprofissional. Segundo Vidal et al. ${ }^{9}$ a falta de uma equipe multiprofissional foi o principal entrave citado pela maioria dos serviços de médio e pequeno portes dos municípios do Estado de Pernambuco para a ação de crescimento e desenvolvimento, o que fez considerar ou classificar um percentual de unidades do estado com esta ação na categoria de "parcialmente implantada".

No grupo infantil, a elevada freqüência de desnutrição leve, sobrepeso/obesidade emer- gindo e a elevada deficiência de micronutrientes (ferro e vitamina A), especialmente no Nordeste 10 , representam argumentos importantes para justificar a inserção do profissional nutricionista nas equipes de atenção à saúde da criança ${ }^{11}$. De Geyndt 12 afirma que a qualidade dos serviços de saúde está relacionada ao acesso às diversas especialidades pela população (multidisciplinaridade do cuidado). O treinamento e a formação de profissionais que lidam com as crianças são fundamentais para atuar dentro de uma percepção holística que abrange o seu compromisso social, relação médico-paciente e uma interação com as necessidades infantis e a realidade local. Segundo o Ministério da Saúde 13, os serviços de saúde às crianças devem estar capacitados para resolução dos problemas de saúde e para atuar contra os fatores indesejáveis do meio ambiente que impedem ou restringem as crianças de atingirem o seu potencial de crescimento e desenvolvimento.

A falta de treinamento tem sido detectada em outros estudos através de deficiências na compreensão dos profissionais sobre o gráfico de crescimento, sobre as intervenções apropriadas 14,15 e sobre o significado do acompanhamento do crescimento 16 . Isso acaba refletindo na falta de orientação às mães sobre o assunto, desconhecimento dos grupos de risco e não realização dos procedimentos que compõem o acompanhamento do crescimento e desenvolvimento. Ratis 16 ao avaliar o acompanhamento do crescimento e desenvolvimento em menores de cinco anos no Estado de Pernambuco, encontrou um deficiente conhecimento dos profissionais sobre a essência do monitoramento, comprovado pelo fato de uma parcela destes profissionais relatarem fazer esta ação apenas quando detectavam algum distúrbio como desnutrição ou diarréia, ou que a mesma deveria ser realizada pela enfermeira na puericultura. E quando se questionou sobre a conduta do profissional ao detectar problemas no crescimento, apenas um terço informou combinar orientação alimentar, aprazamento de consultas e encaminhamento a outro profissional.

A falta de treinamento reflete-se na avaliação do processo de monitorização do crescimento. No estado como um todo, os melhores indicadores do processo não estão muito além de $50 \%$ (pesagem de $53,1 \%$ e peso atual no gráfico de $58,2 \%$ das crianças, informação sobre o comprimento para 53,5\% das mães e pesagem na primeira consulta de 54,3\% das crianças). Acompanhando a deficiência de estrutura, o desempenho, de modo geral, é mais precário nas unidades de saúde do interior do que na Região Metropolitana do Recife. Além disso, a atenção particularizada que 
o programa prevê para crianças com risco nutricional (crianças de baixo peso e peso insuficiente ao nascer; crianças com curva de peso descendente e horizontal; crianças com posição do peso abaixo do percentil 10 e acima de percentil 90) não se concretiza, indistintamente, na Região Metropolitana do Recife ou no interior, denotando falta de foco das ações programáticas.

Em estudo realizado em unidades de saúde do Recife e Olinda, ambos no Estado de Pernambuco, com o objetivo de avaliar a aplicação da estratégia AIDPI pelos agentes comunitários de saúde após treinamento, obteve-se que a avaliação do crescimento foi realizada corretamente, segundo os manuais da AIDPI, por mais de $90 \%$ dos agentes comunitários de saúde 17. Por outro lado, Samico et al. 18, ao avaliar a implantação da atenção à saúde da criança em dois municípios do Estado de Pernambuco, em unidades de saúde do PSF treinadas na estratégia AIDPI, não treinadas e em unidades convencionais, observaram que a ação de crescimento e desenvolvimento apresentou deficiente aplicação nos três tipos de casos, sendo os resultados piores entre os profissionais não treinados e nas unidades convencionais. Em um outro estudo realizado por Amaral et al. 19, em quatro estados do Nordeste, verificou-se que os profissionais que haviam recebido treinamento na AIDPI classificavam corretamente a criança em seu estado nutricional e checavam o peso das crianças com o gráfico do cartão.

Segundo o Ministério da Saúde 2 (p. 27) " $a$ avaliação periódica do ganho de peso permite acompanhar o progresso da criança, identificando aquelas de maior risco de morbimortalidade, sinalizando o alarme precoce para a desnutrição, a causa básica da instalação ou do agravamento da maior parte dos problemas de saúde infantil". Por isso, as consultas são um momento importante para a coleta de medidas antropométricas e registro no gráfico como uma forma mais adequada para acompanhar o crescimento de uma criança nos serviços de saúde.

Contudo, as normas do Ministério da Saúde não estão sendo seguidas nesse aspecto pelas unidades de saúde, visto que cerca de metade das crianças não foi pesada no dia da entrevista. Isso pode ter ocorrido pelo fato de que a maioria das crianças compareceu ao serviço por motivo de doença ou vacinação e um menor percentual por rotina. No entanto, a ocorrência de doença deveria ser um motivo mais que suficiente para a verificação da perda de peso, uma vez que a doença (especialmente as mais freqüentes da infância, diarréia e infecções respiratórias agudas) acarreta inapetência e modificações na alimentação, levando à perda de peso. Na II PESN 5, em 1997, a ocorrência de diarréia nas últimas sema- nas entre as crianças, fez com que mais de $20 \%$ das mães suspendessem a alimentação de seus filhos durante todo o período da enfermidade.

Por outro lado, como a maioria das crianças (40,8\%) compareceu ao serviço para vacinação, esta também deveria ser uma oportunidade para a pesagem e avaliação do crescimento, bem como para a sua captação precoce, visando a um acompanhamento programado do crescimento. No estado, a doença foi o segundo motivo de consulta referido pelas mães, prevalecendo assim, o modelo assistencial tradicionalista e curativo, em que a demanda é espontânea e decorrente dos casos de agravos à saúde. Na Região Metropolitana do Recife, o segundo motivo foi a rotina (visita programada), configurando uma melhor conscientização sobre a importância do acompanhamento periódico das crianças. No interior ainda persiste o modelo curativo e não preventivo das doenças.

Segundo o Ministério da Saúde 2, para uma boa avaliação do crescimento são necessárias pesagens periódicas, obedecendo ao calendário mínimo de consultas para as crianças menores de um ano. Contudo, vale lembrar que já deverá existir um ponto marcado no gráfico do cartão referente ao peso ao nascer. A continuidade no acompanhamento do crescimento constitui característica sine qua non para a efetividade da ação. No presente estudo, observou-se um elevado percentual de crianças que não tinham o peso marcado no gráfico ou que não tinham uma curva de crescimento traçada no cartão. É necessário que os profissionais de saúde estejam sensibilizados e capacitados para conscientizarem as mães da importância de freqüentar continuamente o serviço para uma permanente atenção preventiva.

Contudo, o que se observou foi um desconhecimento da importância do acompanhamento do crescimento, representado pela não marcação do peso no cartão, pela falta de orientação às mães e pelos baixos percentuais de pesagens. Esses aspectos representam uma limitação à efetividade dessa ação de cuidado primário. Segundo Achadi \& Berman 7 (p. 57) “...se as crianças excluídas por causa da irregularidade na assistência são aquelas que de fato mais necessitam serem examinadas, ou seja, as nutricionalmente em risco, um dos objetivos do programa não será atingido".

O baixo peso ao nascer representa o principal indicador da qualidade da assistência à saúde reprodutiva da mulher e um dos melhores indicadores da qualidade de vida das crianças, devido à sua estreita relação com a elevada mortalidade infantil e prejuízos para o seu crescimento linear, ponderal e desenvolvimento mental e motor $20,21,22,23$. Contudo, a falta de registro do peso ao 
nascer no gráfico (apenas metade apresentou esta marcação) evidencia o subestimado papel deste indicador no acompanhamento do estado nutricional no primeiro ano de vida da criança. Por outro lado, Vieira et al. ${ }^{24}$ verificaram que o peso ao nascer foi registrado em $97,2 \%$ dos cartões da criança em estudo realizado no Estado da Bahia com menores de 1 ano em unidades de vacinação. Além disso, o Ministério da Saúde 25 (p. 18) recomenda que "toda criança com história de baixo peso ao nascer deve ser considerada como criança de risco nutricional e acompanhada com mais assiduidade pelos serviços de saúde, principalmente no primeiro ano de vida". Porém, este estudo revelou que o acompanhamento das crianças de baixo peso e peso insuficiente ao nascer foi precário em relação à pesagem, à medida do comprimento, pontuação do peso ao nascer no gráfico e informação às mães, principalmente nas unidades de saúde do interior.

As mães de crianças com direção descendente da curva de crescimento foram pouco orientadas a respeito, com maior prejuízo para as do interior. De acordo com a ação de acompanhamento do crescimento, as crianças com curva de peso descendente ou horizontal devem ser consideradas de risco, independente da posição des-

\section{Resumo}

Realizou-se um diagnóstico situacional da ação de acompanhamento do crescimento em menores de 1 ano da Região Metropolitana do Recife e interior do Estado de Pernambuco, Brasil. Foi utilizado o banco de dados da pesquisa Atenção à Saúde Materno-infantil no Estado de Pernambuco, constituído por 816 crianças e 120 unidades de saúde. Para a análise utilizou-se o Epi Info versão 6.04. Na estrutura dos serviços observou-se que 15,8\% das unidades de saúde não dispunham de balança pesa-bebê nem de Cartão da Criança; 75,4\% não possuíam as normas de acompanhamento do crescimento e desenvolvimento. Dessas variáveis, as duas primeiras revelaram diferença entre as áreas estudadas. Quanto ao processo, $81,2 \%$ das mães dispunham do cartão da criança; $53,1 \%$ das crianças foram pesadas; e apenas $21 \%$ foram medidas em seu comprimento. A orientação dada às mães foi bastante deficitária. Nessas variáveis houve diferença entre as duas áreas. A atenção à criança sob risco nutricional foi pouco considerada nas duas áreas, embora não tenha revelado diferença significante entre interior e região metropolitana. Portanto, a ação de acompanhamento do crescimento não estava efetivamente consolidada no Estado de Pernambuco.

Crescimento; Bem-estar da Criança; Avaliação de Serviços de Saúde ta curva em relação aos percentis. As mães foram pouco informadas sobre os aspetos de acompanhamento do crescimento, especialmente no interior. O que, no entanto, deveria haver uma maior participação das mães e da família no processo de cuidados infantis como principais coresponsáveis pela saúde da criança, e orientação sobre os riscos e conseqüências que o déficit no peso pode trazer para uma criança em fase de crescimento e desenvolvimento acelerado.

O cuidado no primeiro contato funciona como "porta de entrada" para o sistema de saúde e representa uma oportunidade de captação precoce das crianças para o acompanhamento. No entanto, o presente estudo revela que as ações de acompanhamento do crescimento não são realizadas com todas as crianças na primeira consulta, contrariando o recomendado pelo programa. A não realização das atividades mínimas para o acompanhamento do crescimento revela a necessidade de maior prioridade dos gestores sobre esta ação junto às políticas de saúde e educação, especialmente quando se deseja uma gestão plena de atenção básica e quando se busca a efetividade e o impacto do programa sobre o perfil epidemiológico da nutrição na infância, sobre a qualidade de vida e a mortalidade infantil.

\section{Colaboradores}

M. F. Carvalho participou da revisão da literatura, análise e interpretação dos resultados e redação final do artigo. P. I. C. Lira colaborou na análise e interpretação dos resultados, revisão crítica do artigo final e aprovação da versão final para ser publicada. S. A. M. Romani contribuiu na análise e interpretação dos resultados e revisão crítica do artigo final. I. S. Santos participou da revisão crítica do artigo final. A. A. C. A. Veras e M. Batista Filho colaboraram na aquisição dos dados e elaboração da metodologia do artigo.

\section{Agradecimentos}

Às instituições que cederam o banco de dados (Instituto Materno Infantil Professor Fernando Figueira; Departamento de Nutrição da Universidade Federal de Pernambuco; Secretaria Estadual de Saúde de Pernambuco), ao Conselho Nacional de Desenvolvimento Científico e Tecnológico (CNPq) pelas bolsas de produtividade em pesquisa dos professores I. S. Santos, M. Batista Filho e P. I. C. Lira, e à Coordenação de Aperfeiçoamento de Pessoal de Nível Superior (CAPES) pela bolsa de mestrado concedida a M. F. Carvalho. Ao Instituto Nacional de Alimentação e Nutrição, do Ministério da Saúde, pelo financiamento. 


\section{Referências}

1. Corso ACT, Zeni LAZR, Heidrich EMC, Kleine L, Closs G, Botelho LJ, et al. Crianças pré-escolares e o impacto da assistência pública sobre seu crescimento. Revista Ciências da Saúde 1996; 15:29-46.

2. Departamento de Atenção Básica, Secretaria de Políticas de Saúde, Ministério da Saúde. Saúde da criança: acompanhamento do crescimento e desenvolvimento infantil. Brasília: Ministério da Saúde; 2002.

3. Felisberto E, Carvalho EF, Maggi RS, Samico I. Avaliação do processo de implantação da estratégia da Atenção Integrada às Doenças Prevalentes da Infância no Programa de Saúde da Família, no Estado de Pernambuco, Brasil. Cad Saúde Pública 2002; 18:1737-45.

4. Donabedian A. The quality of care: how can it be assessed? JAMA 1988; 260:1743-8.

5. Secretaria de Saúde do Estado de Pernambuco. Atenção à saúde materno-infantil no Estado de Pernambuco. Recife: Editora Bagaço; 2000.

6. Secretaria de Saúde do Estado de Pernambuco. II Pesquisa Estadual de Saúde e Nutrição. Saúde, nutrição, alimentação e condições sócio-econômicas no Estado de Pernambuco. Recife: Governo do Estado de Pernambuco/Instituto Materno-Infantil de Pernambuco/Departamento de Nutrição, Universidade Federal de Pernambuco; 1998.

7. Achadi E, Berman P. Growth monitoring in Indonesia: an assessment of coverage and regularity of attendance, Gedangan village, Central Java, June 1978 November 1981. Food Nutr Bull 1988; 10:56-60.

8. Ghosh S. Successful growth monitoring. Indian Pediatr 1986; 23:759-65.

9. Vidal SA, Frias PG, Marques NM. Avaliação normativa das ações do Programa de Atenção Integral à Saúde da Criança (PAISC) em Pernambuco. Rev Bras Saúde Matern Infant 2001; 1:129-35.

10. Batista Filho M, Rissin A. A transição nutricional no Brasil: tendências regionais e temporais. Cad Saúde Pública 2003; 19 Suppl 1:S181-91.

11. Assis AMO, Santos SMC, Freitas MCS, Santos JM, Silva MCM. O programa saúde da família: contribuições para uma reflexão sobre a inserção do nutricionista na equipe multidisciplinar. Rev Nutr 2002; 15:255-66.

12. De Geyndt W. Five approaches for assessing the quality of care. Hosp Adm (Chic) 1970; 15:21-42.

13. Ministério da Saúde. Assistência integral à saúde da criança: ações básicas. Brasília: Ministério da Saúde; 1984.

14. Gerein N. Is growth monitoring worthwile? Health Policy Plan 1988; 3:181-94.
15. Gerein NM, Ross DA. Is growth monitoring worthwhile? An evaluation of its use in three child health programmes in Zaire. Soc Sci Med 1991; 32:667-75.

16. Ratis CAS. Monitoramento do crescimento e desenvolvimento de crianças atendidas em serviços públicos de saúde do Estado de Pernambuco [Dissertação de Mestrado]. Recife: Departamento de Nutrição, Universidade Federal de Pernambuco; 2003.

17. Vidal SA, Silva EV, Oliveira MG, Siqueira AM, Felisberto E, Samico I, et al. Avaliação da aplicação da estratégia Atenção Integral às Doenças Prevalentes da Infância (AIDPI) por agentes comunitários de saúde. Rev Bras Saúde Matern Infant 2003; 3:205-13.

18. Samico I, Hartz ZMA, Felisberto E, Carvalho EF. Atenção à saúde da criança: uma análise do grau de implantação e da satisfação de profissionais e usuários em dois municípios do Estado de Pernambuco, Brasil. Rev Bras Saúde Matern Infant 2005; 5:229-40.

19. Amaral J, Gouws E, Bryce J, Leite AJM, Cunha ALA, Victora CG. Effect of Integrated Management of Childhood Illness (IMCI) on health worker performance in Northeast-Brazil. Cad Saúde Pública 2004; 20 Suppl 2:S209-19.

20. Lira PIC, Ashworth A, Morris SS. Low birth weight and morbidity from diarrhea and respiratory infection in Northeast, Brazil. J Pediatr 1996; 128:497-504.

21. Eickmann SH, Lira PIC, Lima MC. Desenvolvimento mental e motor aos 24 meses de crianças nascidas a termo com baixo peso. Arq Neuropsiquiatr 2002; 60:748-54.

22. Romani SAM. Perfil nutricional de crianças da Zona da Mata Meridional de Pernambuco. Nordeste do Brasil: um estudo de coorte [Tese de Doutorado]. Recife: Departamento de Nutrição, Universidade Federal de Pernambuco; 2003.

23. Victora CG, Barros FC, Vaughan JP, Martines JC, Beria JU. Birthweight, socioeconomic status and growth of Brazilian infants. Ann Hum Biol 1987; 14:49-57.

24. Vieira GO, Vieira TO, Costa COM, Santana-Netto PV, Cabral VA. Uso do cartão da criança em Feira de Santana, Bahia. Rev Bras Saúde Matern Infant 2005; 5:177-84.

25. Ministério da Saúde. Fundamentos técnico-científicos e orientações práticas para o acompanhamento do crescimento e desenvolvimento. Parte 1: crescimento. 1ạ Ed. Brasília: Ministério da Saúde; 2001.

Recebido em 20/Jul/2006

Versão final reapresentada em 12/Jun/2007

Aprovado em 29/Jun/2007 